\title{
Three-Body Forces in Multiparticle Dynamics: New Sites of Fundamental Dynamics. ${ }^{1}$
}

\author{
A.A. Arkhipov \\ State Research Center "Institute for High Energy Physics" \\ 142280 Protvino, Moscow Region, Russia
}

\begin{abstract}
A manifestation of the three-body forces in multiparticle dynamics is discussed. The minireview of our recent results has been presented.
\end{abstract}

\section{Introduction}

Three-body forces are fundamental forces which take place in the multiparticle systems where the number of particles is greater than two. The simplest one of such systems is a three-particle system. However, it's clear that in any multiparticle system there are two-body or pair interactions between particles and the question arises how could we separate three-body forces from two-body ones. Of course, a definition of three-body forces depends on a formalism which one uses. It turns out that single-time formalism in Quantum Field Theory is a useful tool to make it.

Using the LSZ or Bogoljubov reduction formulae in Quantum Field Theory we can easily obtain the following cluster structure for $3 \rightarrow 3$ scattering amplitude

$$
\mathcal{F}_{123}=\mathcal{F}_{12}+\mathcal{F}_{23}+\mathcal{F}_{13}+\mathcal{F}_{123}^{C}
$$

where $\mathcal{F}_{i j},(i, j=1,2,3, i \neq j)$ are $2 \rightarrow 2$ scattering amplitudes, $\mathcal{F}_{123}^{C}$ is called the connected part of the $3 \rightarrow 3$ scattering amplitude.

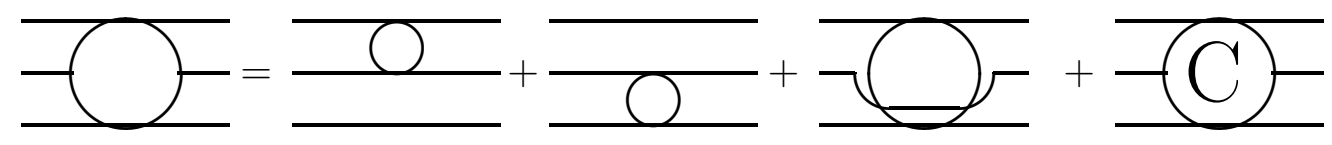

In the framework of the single-time formalism in Quantum Field Theory developed in [1] we construct the $3 \rightarrow 3$ off energy shell scattering amplitude $T_{123}(E)$ with the same (cluster) structure

$$
T_{123}(E)=T_{12}(E)+T_{23}(E)+T_{13}(E)+T_{123}^{C}(E) .
$$

The three particle interaction quasipotential $V_{123}(E)$ is related to the off energy shell $3 \rightarrow 3$ scattering amplitude $T_{123}(E)$ by the Lippmann-Schwinger transformation (LStransformation)

$$
T_{123}(E)=V_{123}(E)+V_{123}(E) G_{0}(E) T_{123}(E) .
$$

\footnotetext{
${ }^{1}$ The talk presented at XXXII International Symposium on Multiparticle Dynamics, Alushta, Crimea, Ukraine, September 7-12, 2002.
} 
There exists the same LS-transformation between two particle interaction quasipotentials $V_{i j}$ and off energy shell $2 \rightarrow 2$ scattering amplitudes $T_{i j} \quad(i, j=1,2,3, i \neq j)$

$$
T_{i j}(E)=V_{i j}(E)+V_{i j}(E) G_{0}(E) T_{i j}(E) .
$$

In Quantum Field Theory a three particle interaction quasipotential has the structure [2]

$$
V_{123}(E)=V_{12}(E)+V_{23}(E)+V_{13}(E)+V_{0}(E),
$$

where $V_{0}(E)$ is the three-body forces quasipotential; it represents the defect of three particle interaction quasipotential over the sum of two particle quasipotentials and describes the true three-body interactions. Three-body forces quasipotential is an inherent connected part of total three particle interaction quasipotential which cannot be represented by the sum of pair quasipotentials. The three-body forces could be seen even in the perturbation theory expansions. However, and we would like to stress it, formulae (14) provide a constructive definition of the three-body forces in the framework of local Quantum Field Theory without having the use of perturbation theory.

The three-body forces scattering amplitude is related to the three-body forces quasipotential by the LS-transformation

$$
T_{0}(E)=V_{0}(E)+V_{0}(E) G_{0}(E) T_{0}(E)
$$

It should be pointed out once more that the three-body forces appear as a result of consistent consideration of the three-body problem in the framework of local Quantum Field Theory.

\section{Global analyticity of the three-body forces}

Let us introduce the following useful notations:

$$
\begin{gathered}
<p_{1}^{\prime} p_{2}^{\prime} p_{3}^{\prime}|S-1| p_{1} p_{2} p_{3}>=2 \pi i \delta^{4}\left(\sum_{i=1}^{3} p_{i}^{\prime}-\sum_{j=1}^{3} p_{j}\right) \mathcal{F}_{123}\left(s ; \hat{e}^{\prime}, \hat{e}\right), \\
s=\left(\sum_{i=1}^{3} p_{i}^{\prime}\right)^{2}=\left(\sum_{j=1}^{3} p_{j}\right)^{2} .
\end{gathered}
$$

$\hat{e}^{\prime}, \hat{e} \in S_{5}$ are two unit vectors on five-dimensional sphere describing the configuration of three-body system in initial and final states (before and after scattering). We will denote the quantity $T_{0}$ restricted on the energy shell as

$$
\left.T_{0}\right|_{\text {on energy shell }}=\mathcal{F}_{0} \text {. }
$$

The unitarity condition for the quantity $\mathcal{F}_{0}$ with account for the introduced notations can be written in form [3, 4 ]

$$
\begin{gathered}
\operatorname{Im} \mathcal{F}_{0}\left(s ; \hat{e}^{\prime}, \hat{e}\right)=\pi A_{3}(s) \int d \Omega_{5}\left(\hat{e}^{\prime \prime}\right) \mathcal{F}_{0}\left(s ; \hat{e}^{\prime}, \hat{e}^{\prime \prime}\right) \mathcal{F}_{0}^{*}\left(s ; \hat{e}, \hat{e}^{\prime \prime}\right)+H_{0}\left(s ; \hat{e}^{\prime}, \hat{e}\right) \\
\operatorname{Im} \mathcal{F}_{0}\left(s ; \hat{e}^{\prime}, \hat{e}\right) \equiv \frac{1}{2 i}\left[\mathcal{F}_{0}\left(s ; \hat{e}^{\prime}, \hat{e}\right)-\mathcal{F}_{0}\left(s ; \hat{e}, \hat{e}^{\prime}\right)\right]
\end{gathered}
$$


where

$$
A_{3}(s)=\Gamma_{3}(s) / S_{5},
$$

$\Gamma_{3}(s)$ is the three-body phase-space volume, $S_{5}$ is the volume of unit five-dimensional sphere, $H_{0}$ defines the contribution of all inelastic channels emerging due to three-body forces.

Let us introduce a special notation for the scalar product of two unit vectors $\hat{e}^{\prime}$ and $\hat{e}$

$$
\cos \omega=\hat{e}^{\prime} \cdot \hat{e}
$$

The other notation for the three-body forces scattering amplitude will be used as well

$$
\mathcal{F}_{0}\left(s ; \hat{e}^{\prime}, \hat{e}\right)=\mathcal{F}_{0}(s ; \eta, \cos \omega),
$$

where all other variables are denoted through $\eta$. Now we are able to formulate our basic assumption concerning the analytical properties of the three-body forces scattering amplitude [3, 4].

We will assume that for physical values of the variable $s$ and fixed values of $\eta$ the amplitude $\mathcal{F}_{0}(s ; \eta, \cos \omega)$ is an analytical function of the variable $\cos \omega$ in the ellipse $E_{0}(s)$ with the semi-major axis

$$
z_{0}(s)=1+\frac{M_{0}^{2}}{2 \Pi^{2}(s)}
$$

and for any $\cos \omega \in E_{0}(s)$ and physical values of $\eta$ it is polynomially bounded in the variable $s . M_{0}$ is some constant having mass dimensionality.

Such analyticity of the three-body forces amplitude was called a global one. The global analyticity may be considered as a direct geometric generalization of the known analytical properties of two-body scattering amplitude strictly proved in the local quantum field theory. At the same time the global analyticity results in the generalized asymptotic bounds.

\section{GLOBAL ANALYTICITY \& UNITARITY \\ $\Downarrow$ \\ GENERALIZED ASYMPTOTIC BOUNDS}

For example, the generalized asymptotic bound for $O(6)$-invariant three-body forces scattering amplitude looks like [3, 4,

$$
\operatorname{Im} \mathcal{F}_{0}(s ; \cos \omega=1) \leq \text { Const } s^{3 / 2}\left(\frac{\ln s / s_{0}^{\prime}}{M_{0}}\right)^{5}=\text { Const } s^{3 / 2} R_{0}^{5}(s),
$$

where $R_{0}(s)$ is the effective radius of the three-body forces

$$
R_{0}(s)=\frac{\Lambda_{0}}{\Pi(s)}=\frac{r_{0}}{M_{0}} \ln \frac{s}{s_{0}^{\prime}}, \quad \Pi(s)=\frac{\sqrt{s}}{2}, \quad s \rightarrow \infty,
$$

$r_{0}$ is defined by the power of growth of the amplitude $\mathcal{F}_{0}$ at high energies 4], $M_{0}$ defines the semi-major axis of the global analyticity ellipse (91), $\Lambda_{0}$ is the effective global orbital momentum, $\Pi(s)$ is the global momentum of three-body system, $s_{0}^{\prime}$ is a scale defining a range of unitarity saturation of three-body forces. 
If we want to have a possibility for an experimental verification of generalized asymptotic bound (10) we have to establish a connection between the three-body forces scattering amplitude and the experimentally measurable quantities. In fact, we found the connection of the three-body forces scattering amplitude with the experimentally measurable quantity which is the total cross section in scattering from deuteron [5, 6], and the relation of the three-body forces scattering amplitude to one-particle inclusive cross sections was established as well [7].

Now we shall briefly sketch the basic results of our analysis.

\section{Three-body forces in single diffraction dissociation}

The formula relating one-particle inclusive cross-section with the imaginary part of the three-body forces scattering amplitude looks like [6, 7]

$$
\begin{aligned}
& 2 E_{N}(\vec{\Delta}) \frac{d \sigma_{h N \rightarrow N X}}{d \vec{\Delta}}(s, \vec{\Delta})=-\frac{(2 \pi)^{3}}{I(s)} \operatorname{Im} \mathcal{F}_{0}^{s c r}(\bar{s} ;-\vec{\Delta}, \vec{\Delta}, \vec{q} ; \vec{\Delta},-\vec{\Delta}, \vec{q}) \text {, } \\
& \operatorname{Im} \mathcal{F}_{0}^{s c r}(\bar{s} ;-\vec{\Delta}, \vec{\Delta}, \vec{q} ; \vec{\Delta},-\vec{\Delta}, \vec{q})=\operatorname{Im} \mathcal{F}_{0}(\bar{s} ;-\vec{\Delta}, \vec{\Delta}, \vec{q} ; \vec{\Delta},-\vec{\Delta}, \vec{q})- \\
& -4 \pi \int d \vec{\Delta}^{\prime} \frac{\delta\left[E_{N}\left(\vec{\Delta}-\vec{\Delta}^{\prime}\right)+\omega_{h}\left(\vec{q}+\vec{\Delta}^{\prime}\right)-E_{N}(\vec{\Delta})-\omega_{h}(\vec{q})\right]}{2 \omega_{h}\left(\vec{q}+\vec{\Delta}^{\prime}\right) 2 E_{N}\left(\vec{\Delta}-\vec{\Delta}^{\prime}\right)} \times \\
& \operatorname{Im} \mathcal{F}_{h N}\left(\hat{s} ; \vec{\Delta}, \vec{q} ; \vec{\Delta}-\vec{\Delta}^{\prime}, \vec{q}+\vec{\Delta}^{\prime}\right) \operatorname{Im} \mathcal{F}_{0}\left(\bar{s} ;-\vec{\Delta}, \vec{\Delta}-\vec{\Delta}^{\prime}, \vec{q}+\vec{\Delta}^{\prime} ; \vec{\Delta},-\vec{\Delta}, \vec{q}\right), \\
& E_{N}(\vec{\Delta})=\sqrt{\vec{\Delta}^{2}+M_{N}^{2}}, \quad \omega_{h}(\vec{q})=\sqrt{\vec{q}^{2}+m_{h}^{2}}, \quad I(s)=2 \lambda^{1 / 2}\left(s, m_{h}^{2}, M_{N}^{2}\right), \\
& \hat{s}=\frac{\bar{s}+m_{h}^{2}-2 M_{N}^{2}}{2}, \quad \bar{s}=2\left(s+M_{N}^{2}\right)-M_{X}^{2}, \quad t=-4 \vec{\Delta}^{2} .
\end{aligned}
$$

I'd like to draw attention to the minus sign in the R.H.S. of Eq. (12). Because we have a positive physical quantity in the L.H.S. this means that second term in the R.H.S. of Eq. (13) has to be dominated over the first one. It's really true. The simple model for the three-body forces

$$
\operatorname{Im} \mathcal{F}_{0}\left(s ; \vec{p}_{1}, \vec{p}_{2}, \vec{p}_{3} ; \vec{q}_{1}, \vec{q}_{2}, \vec{q}_{3}\right)=f_{0}(s) \exp \left\{-\frac{R_{0}^{2}(s)}{4} \sum_{i=1}^{3}\left(\vec{p}_{i}-\vec{q}_{i}\right)^{2}\right\}
$$

where $f_{0}(s), R_{0}(s)$ are model parametric functions of $s$, gives the following result for the one-particle inclusive cross section in the region of diffraction dissociation

$$
\begin{aligned}
\frac{s}{\pi} \frac{d \sigma_{h N \rightarrow N X}}{d t d M_{X}^{2}} & =\frac{(2 \pi)^{3}}{I(s)} \chi(\bar{s}) \operatorname{Im} \mathcal{F}_{0}(\bar{s} ;-\vec{\Delta}, \vec{\Delta}, \vec{q} ; \vec{\Delta},-\vec{\Delta}, \vec{q}) \\
& =\frac{(2 \pi)^{3}}{I(s)} \chi(\bar{s}) f_{0}(\bar{s}) \exp \left[\frac{R_{0}^{2}(\bar{s})}{2} t\right]
\end{aligned}
$$

where

$$
\chi(\bar{s})=\frac{\sigma_{h N}^{t o t}(\bar{s} / 2)}{2 \pi\left[B_{h N}(\bar{s} / 2)+R_{0}^{2}(\bar{s})\right]}-1
$$


The function $\chi$ has a clear physical meaning: This function originates from initial and final states interactions and describes the shadowing (eclipsing) effect or the effect of screening the three-body forces by two-body ones [7].

If we take the usual parametrization for one-particle inclusive cross-section in the region of diffraction dissociation

$$
\frac{s}{\pi} \frac{d \sigma}{d t d M_{X}^{2}}=A\left(s \cdot M_{X}^{2}\right) \exp \left[b\left(s, M_{X}^{2}\right) t\right]
$$

then one obtains for the quantities $A$ and $b$

$$
A\left(s, M_{X}^{2}\right)=\frac{(2 \pi)^{3}}{I(s)} \chi(\bar{s}) f_{0}(\bar{s}), \quad b\left(s, M_{X}^{2}\right)=\frac{R_{0}^{2}(\bar{s})}{2} .
$$

Eq. (17) displays a remarkable fact:

the effective radius of three-body forces is related to the slope of diffraction cone for inclusive diffraction dissociation processes in the same way as the effective radius of two-body forces is related to the slope of diffraction cone in elastic scattering processes.

Moreover, from the expressions

$$
R_{0}(\bar{s})=\frac{r_{0}}{M_{0}} \ln \bar{s} / s_{0}^{\prime}, \quad \bar{s}=2\left(s+M_{N}^{2}\right)-M_{X}^{2}
$$

it follows that

the slope of diffraction cone for inclusive diffraction dissociation processes at fixed energy decreases with the growth of missing mass and increases with the growth of energy at fixed value of missing mass.

This property agrees well qualitatively with the experimentally observable picture. Actually, we have even a more remarkable fact: Shrinkage or narrowing of diffraction cone for inclusive diffraction dissociation processes with the growth of energy at a fixed missing mass and widening of this cone with the growth of missing mass at a fixed energy is of universal character. As it follows from Eq. (15) this property is the consequence of the fact that the one-particle inclusive cross-section up to flux factor depends on the variables $s$ and $M_{X}^{2}$ via one variable $\bar{s}$ which is a linear combination of $s$ and $M_{X}^{2}$. This peculiar "scaling" is the manifestation of $O(6)$-symmetry of the three-body forces. It would be very desirable to experimentally study this new scaling law related to the symmetry of the new fundamental (three-body) forces.

There is a very important relation

$$
A\left(s, M_{X}^{2}\right)=\frac{\bar{s} M_{N}\left[R_{0}^{2}(\bar{s})+R_{d}^{2}\right]^{3 / 2}}{(2 \pi)^{3 / 2} I(s)} \delta \sigma^{\text {inel }}(\bar{s}) \text {. }
$$

Eq. (18) establishes a deep connection of inelastic shadow correction in scattering from deuteron with one-particle inclusive cross-section. This relation for one's turn allowed us to express the inelastic shadow correction via a total single diffractive dissociation cross-section [8]. This will be shown in the next section. 


\section{Three-body forces in scattering from deuteron}

We have applied our approach to study a shadow dynamics in scattering from deuteron in some details. In this way a new simple formula for the shadow corrections to the total cross-section in scattering from deuteron has been derived and new scaling characteristics with a clear physical interpretation have been established. Here we shall briefly sketch the basic results of our analysis of high-energy particle scattering from deuteron. As has been shown in [8], the total cross-section in the scattering from deuteron can be expressed by the formula

$$
\sigma_{h d}^{t o t}(s)=\sigma_{h p}^{t o t}(s)+\sigma_{h n}^{t o t}(s)-\delta \sigma(s),
$$

where $\sigma_{h d}, \sigma_{h p}, \sigma_{h n}$ are the total cross-sections in scattering from deuteron, proton and neutron,

$$
\begin{gathered}
\delta \sigma(s)=\delta \sigma^{e l}(s)+\delta \sigma^{i n e l}(s)=2 \sigma^{e l}(s) a^{e l}\left(x_{e l}\right)+2 \sigma_{s d}^{e x}(s) a^{\text {inel }}\left(x_{\text {inel }}\right), \\
\sigma^{e l}(s) \equiv \frac{\sigma_{h N}^{\text {tot } 2}(s)}{16 \pi B_{e l}(s)}, \quad a^{e l}\left(x_{e l}\right)=\frac{x_{e l}^{2}}{1+x_{e l}^{2}}, \quad x_{e l}^{2} \equiv \frac{2 B_{e l}(s)}{R_{d}^{2}}=\frac{R_{2}^{2}(s)}{R_{d}^{2}}, \\
a^{i n e l}\left(x_{\text {inel }}\right)=\frac{x_{\text {inel }}^{2}}{\left(1+x_{\text {inel }}^{2}\right)^{3 / 2}}, \quad x_{\text {inel }}^{2} \equiv \frac{R_{3}^{2}(s)}{R_{d}^{2}}=\frac{2 B_{s d}(s)}{R_{d}^{2}},\left.\quad B_{s d}(s) \equiv b\left(s, M_{X}^{2}\right)\right|_{M_{X}^{2}=2 M_{N}^{2} .}
\end{gathered}
$$

The total single diffractive dissociation cross-section $\sigma_{s d}^{e x}(s)$ is defined by the following equation 8

$$
\sigma_{s d}^{\varepsilon}(s)=\pi \int_{M_{\min }^{2}}^{\varepsilon s} \frac{d M_{X}^{2}}{s} \int_{t_{-}\left(M_{X}^{2}\right)}^{t_{+}\left(M_{X}^{2}\right)} d t \frac{d \sigma}{d t d M_{X}^{2}},
$$

where

$$
\varepsilon=\varepsilon^{e x}=\sqrt{2 \pi} / 2 M_{N} R_{d},
$$

and we supposed that $\sigma_{h p}^{t o t}=\sigma_{h n}^{t o t}=\sigma_{h N}^{t o t}$ and $B_{e l}^{h p}=B_{e l}^{h n}=B_{e l}$ at high energies. The first term in the R.H.S. of Eq. (19) generalizes the known Glauber correction

$$
\delta \sigma^{e l}(s)=\delta \sigma_{G}(s)=\frac{\sigma_{h N}^{t o t} 2(s)}{4 \pi R_{d}^{2}}, \quad x_{e l}^{2}<<1,
$$

but the second term in the R.H.S. of Eq. (19) is totally new and comes from the contribution of the three-body forces to the hadron-deuteron total cross section.

The expressions for the shadow corrections have quite a transparent physical meaning, both elastic $a^{e l}$ and inelastic $a^{\text {inel }}$ scaling functions related to elastic and inelastic parts of the total shadow correction have a clear physical interpretation [9]. The function $a^{e l}$ measures out a portion of elastic rescattering events among of all the events during the interaction of an incident particle with a deuteron as a whole, and this function attached to the total probability of elastic interaction of an incident particle with a separate nucleon in a deuteron. Correspondingly, the function $a^{\text {inel }}$ measures out a portion of inelastic events of inclusive type among of all the events during the interaction of an incident particle with a deuteron as a whole, and this function attached to the total probability of single diffraction dissociation of an incident particle on a separate nucleon in a deuteron. The scaling variables $x_{e l}$ and $x_{\text {inel }}$ have quite a clear physical meaning too. The dimensionless quantity $x_{e l}$ characterizes the effective distances measured in the units of "fundamental length", which the deuteron size is, in elastic interactions, but the similar quantity $x_{\text {inel }}$ 
characterizes the effective distances measured in the units of the same "fundamental length" during inelastic interactions.

The functions $a^{e l}$ and $a^{\text {inel }}$ have a different behaviour: $a^{e l}$ is a monotonic function while $a^{\text {inel }}$ has the maximum at the point $x_{\text {inel }}^{\text {max }}=\sqrt{2}$ where $a^{\text {inel }}\left(x_{\text {inel }}^{\text {max }}\right)=2 / 3 \sqrt{3}$. The existence of the maximum in the function $a^{\text {inel }}$ results an interesting physical effect of weakening the inelastic eclipsing (screening) at superhigh energies. The energy $s_{m}$ at the maximum of $a^{\text {inel }}$ can be calculated from the equation $R_{3}^{2}\left(s_{m}\right)=2 R_{d}^{2}$, and one obtains in this way: $\sqrt{s_{m}}=9.0110^{8} \mathrm{GeV}=901 \mathrm{PeV}$ [8, 9].

From geometrical point of view, if we would consider the standard Glauber correction as the shadowing where one two-dimensional sphere is in eclipse with the other twodimensional sphere, then the inelastic screening and the effect of its weakening should be considered as the shadowing where a five-dimensional sphere "casts" its shadow on a two-dimensional sphere. So, we really see here the true play of the spheres.

It is very important that formula (19) contains only experimentally measured quantities. Therefore we can verify the new structure for the shadow corrections in elastic scattering from deuteron using the existing experimental data on proton-deuteron and antiproton-deuteron total cross sections and experimental data on single diffractive dissociation in scattering from nucleon. The results of comparison are shown in Figs. 1-2 extracted from paper [8].

We would like to emphasize that in the fit to the data on antiproton-deuteron total cross sections our global fit to the data on antiproton-proton total cross section [10] has been used (see next section), and $R_{d}^{2}$ was considered as a single free fit parameter. After that a comparison with the data on proton-deuteron total cross sections has been made without any free parameters: $R_{d}^{2}$ was fixed by the previous fit to the data on antiprotondeuteron total cross sections, our fit yielded $R_{d}^{2}=66.61 \pm 1.16 \mathrm{GeV}^{-2}$, and our global fit to the data on proton-proton total cross section [10] has been used as well (see next section). If we take into account the latest experimental value for the deuteron matter radius $r_{d, m}=1.963(4) \mathrm{fm}$ [11] then we can find that the fitted value for the $R_{d}^{2}$ satisfies with a good accuracy the equality [8, 9]

$$
R_{d}^{2}=\frac{2}{3} r_{d, m}^{2}, \quad\left(r_{d, m}^{2}=3.853 \mathrm{fm}^{2}=98.96 \mathrm{GeV}^{-2}\right)
$$

\section{$5 \quad$ Global structure of $\bar{p} p$ and $p p$ total cross sections}

Recently [7, 10, a simple theoretical formula describing the global structure of $p p$ and $p \bar{p}$ total cross-sections in the whole range of energies available up today has been derived by an application of single-time formalism in QFT and general theorems a là Froissart. The fit to the experimental data with the formula was made, and it was shown that there is a very good correspondence of the theoretical formula to the existing experimental data obtained at the accelerators. Moreover, it turned out [12 there is a very good correspondence of the theory to all existing cosmic ray experimental data as well: The predicted values for $\sigma_{p p}^{\text {tot }}$ obtained from theoretical description of all existing accelerators data are completely compatible with the values obtained from cosmic ray experiments 13. The global structure of (anti)proton-proton total cross section is shown in Figs. 3-4 extracted from papers [10, 12].

The theoretical formula describing the global structure of (anti)proton-proton total cross section has the following structure [10] 


$$
\sigma_{(\bar{p}) p p}^{t o t}(s)=\sigma_{a s m p t}^{t o t}(s)\left[1+\chi_{(\bar{p}) p p}(s)\right]
$$

where

$$
\begin{gathered}
\sigma_{\text {asmpt }}^{\text {tot }}(s)=2 \pi\left[B_{e l}(s)+(1-\beta) R_{3}^{2}(s)\right]=\left[42.0479+1.7548 \ln ^{2}(\sqrt{s} / 20.74)\right](m b), \\
B_{e l}(s)=R_{2}^{2}(s) / 2=\left[11.92+0.3036 \ln ^{2}(\sqrt{s} / 20.74]\left(G e V^{-2}\right),\right. \\
\left.R_{3}^{2}(s)\right|_{\beta<<1}=\left[0.40874044 \sigma_{\text {asmpt }}^{\text {tot }}(s)(m b)-B_{e l}(s)\right]\left(G e V^{-2}\right)= \\
=\left[5.267+0.4137 \ln ^{2} \sqrt{s} / 20.74\right]\left(G e V^{-2}\right) \\
\beta=\frac{x_{\text {inel }}^{2}}{4\left(1+x_{\text {inel }}^{2}\right)}, \quad x_{\text {inel }}^{2}=\frac{R_{3}^{2}(s)}{R_{d}^{2}}=\frac{2 B_{s d}(s)}{R_{d}^{2}}
\end{gathered}
$$

$B_{e l}(s)$ is the slope of nucleon-nucleon differential elastic scattering cross section, $R_{2}(s)$ is the effective radius of two-nucleon forces, $R_{3}(s)$ is the effective radius of three-nucleon forces, $R_{d}$ characterizes the internucleon distance in a deuteron, the functions $\chi_{(\bar{p}) p p}(s)$ describe low-energy parts of (anti)proton-proton total cross sections and asymptotically tend to zero at $s \rightarrow \infty$ (see details in the original paper [10])

$$
\begin{gathered}
\chi_{\bar{p} p}(s)=\frac{c}{\sqrt{s-4 m_{N}^{2}} R_{0}^{3}(s)}\left(1+\frac{d_{1}}{\sqrt{s}}+\frac{d_{2}}{s}+\frac{d_{3}}{s^{3 / 2}}\right), \\
d_{1}=(-12.12 \pm 1.023) G e V, \quad d_{2}=(89.98 \pm 15.67) G e V^{2} \\
d_{3}=(-110.51 \pm 21.60) G e V^{3}, \quad c=(6.655 \pm 1.834) G e V^{-2} .
\end{gathered}
$$

and

$$
\begin{gathered}
\chi_{p p}(s)=\left.\left(\frac{c_{1}}{\sqrt{s-4 m_{N}^{2}} R_{0}^{3}(s)}-\frac{c_{2}}{\sqrt{s-s_{t h r}} R_{0}^{3}(s)}\right)(1+d(s))\right|_{s>s_{t h r}}+\operatorname{Res}(s), \\
c_{1}=(192.85 \pm 1.68) G e V^{-2}, \quad c_{2}=(186.02 \pm 1.67) G e V^{-2} \\
\left.d(s)=\sum_{k=1}^{8} \frac{d_{k}}{s^{k / 2}}, \quad \operatorname{Res}(s)=\sum_{i=1}^{N} \frac{C_{R}^{i} s_{R}^{i} \Gamma_{R}^{i 2}}{\sqrt{s\left(s-4 m_{N}^{2}\right)}\left[\left(s-s_{R}^{i}\right)^{2}+s_{R}^{i} \Gamma_{R}^{i}{ }^{2}\right.}\right] \\
s_{t h r}=(3.5283 \pm 0.0052) G e V^{2} .
\end{gathered}
$$

For the numerical values of the parameters $d_{i}(i=1, \ldots 8)$ see original paper [10].

The formula (23) represents the total cross section in a factorized form. One factor describes high energy asymptotics of total cross section and it has the universal energy dependence predicted by the general theorems in local Quantum Field Theory (Froissart theorem). The other factor is responsible for the behaviour of total cross section at low energies and it has a complicated resonance structure. However this factor has also the universal asymptotics at elastic threshold. It is a remarkable fact that the low energy part of total cross section has been derived by application of the generalized Froissart theorem for a three-body forces scattering amplitude. 
The nontrivial feature of the formula for the proton-proton total cross section is the presence of the new "threshold" $s_{t h r}=3.5283 \mathrm{GeV}^{2}$ which is near the elastic one. Moreover, Eq. (24) shows that geometrical scaling in a naive form $\sigma_{a s m p t}^{\text {tot }}(s)=\operatorname{Const} B_{e l}(s)$ is not valid. However, from Eq. (24) it follows the generalized geometrical scaling which looks like

$$
\sigma_{\text {asmpt }}^{\text {tot }}(s)=2 \pi B_{e l}(s)[1+2 \gamma(1-\beta)]
$$

where $\beta$ is defined above and

$$
\gamma=\frac{R_{3}^{2}(s)}{2 B_{e l}(s)}=\frac{R_{3}^{2}(s)}{R_{2}^{2}(s)}=\frac{B_{s d}(s)}{B_{e l}(s)} .
$$

Some information concerning the diproton resonances is collected in Table 1 . The positions of resonances and their widths, listed in Table 1, were fixed in our fit, and only relative contributions of the resonances $C_{R}^{i}$ have been considered as free fit parameters. Fitted parameters $C_{R}^{i}$ obtained by the fit are listed in Table 1 too.

Our fitting curve concerning low-energy region is shown in Fig. 5. We also plotted in Fig. 6 the resonance structure of proton-proton total cross section at low energies without the experimental points but with dashed line corresponding the "background" where all resonances are switched off. As it is seen from this Figure there is a clear signature for the diproton resonances. We may conclude that the diproton resonances are confirmed by the data set for proton-proton total cross section at low energies from statistical point of view by the good fit [16].

From the global structure of proton-proton total cross-section it follows that the new "threshold", which is near the elastic one, looks like a manifestation of a new unknown particle:

$$
\sqrt{s_{t h r}}=2 m_{p}+m_{\mathcal{L}}, \quad m_{\mathcal{L}}=1.833 \mathrm{MeV}
$$

Table 1. Diproton resonances.

\begin{tabular}{|l|c|r|r|}
\hline$m_{R}(\mathrm{MeV})$ & $\Gamma_{R}(\mathrm{MeV})$ & Refs. & $C_{R}\left(\mathrm{GeV}^{2}\right)$ \\
\hline $1937 \pm 2$ & $7 \pm 2$ & {$[14]$} & $0.058 \pm 0.018$ \\
$1947(5) \pm 2.5$ & $8 \pm 3.9$ & {$[15]$} & $0.093 \pm 0.028$ \\
$1955 \pm 2$ & $9 \pm 4$ & {$[14]$} & $0.158 \pm 0.024$ \\
$1965 \pm 2$ & $6 \pm 2$ & {$[14]$} & $0.138 \pm 0.009$ \\
$1980 \pm 2$ & $9 \pm 2$ & {$[14]$} & $0.310 \pm 0.051$ \\
$1999 \pm 2$ & $9 \pm 4$ & {$[14]$} & $0.188 \pm 0.070$ \\
$2008 \pm 3$ & $4 \pm 2$ & {$[14]$} & $0.176 \pm 0.050$ \\
$2027 \pm ?$ & $10-12$ & {$[16]$} & $0.121 \pm 0.018$ \\
$2087 \pm 3$ & $12 \pm 7$ & {$[14]$} & $-0.069 \pm 0.010$ \\
$2106 \pm 2$ & $11 \pm 5$ & {$[14]$} & $-0.232 \pm 0.025$ \\
$2127(9) \pm 5$ & $4 \pm 2$ & {$[14]$} & $-0.222 \pm 0.056$ \\
$2180(72) \pm 5$ & $7 \pm 3$ & {$[14]$} & $0.131 \pm 0.015$ \\
$2217 \pm ?$ & $8-10$ & {$[16]$} & $0.112 \pm 0.031$ \\
$2238 \pm 3$ & $22 \pm 8$ & {$[14]$} & $0.221 \pm 0.078$ \\
$2282 \pm 4$ & $24 \pm 9$ & {$[14]$} & $0.098 \pm 0.024$ \\
\hline
\end{tabular}

This particle was called [16] as $\mathcal{L}$-particle from the word lightest. It should be emphasized that we predicted the position of the new "threshold" with a high accuracy. Of course, 
the natural questions have been arisen. What is the physical nature and dynamical origin of $\mathcal{L}$-particle? Could $\mathcal{L}$-particle be related to the experimentally observed diproton resonances spectrum? We present the answers to these questions in the next section.

\section{$6 \quad \mathcal{L}$-particle and Kaluza-Klein world}

The original idea of Kaluza and Klein is based on the hypothesis that the input space-time is a $(4+d)$-dimensional space $\mathcal{M}_{(4+d)}$ which can be represented as a tensor product of the visible four-dimensional world $M_{4}$ with a compact internal $d$-dimensional space $K_{d}$

$$
\mathcal{M}_{(4+d)}=M_{4} \times K_{d}
$$

The compact internal space $K_{d}$ is space-like one i.e. it has only spatial dimensions which may be considered as extra spatial dimensions of $M_{4}$. In according with the tensor product structure of the space $\mathcal{M}_{(4+d)}$ the metric may be chosen in a factorizable form. This means that if $z^{M}=\left\{x^{\mu}, y^{m}\right\},(M=0,1, \ldots, 3+d, \mu=0,1,2,3, m=1,2, \ldots, d)$, are local coordinates on $\mathcal{M}_{(4+d)}$ then the factorizable metric looks like

$$
d s^{2}=\mathcal{G}_{M N}(z) d z^{M} d z^{N}=g_{\mu \nu}(x) d x^{\mu} d x^{\nu}+\gamma_{m n}(x, y) d y^{m} d y^{n}
$$

where $g_{\mu \nu}(x)$ is the metric on $M_{4}$.

In the year 1921, Kaluza proposed a unification of the Einstein gravity and the Maxwell theory of electromagnetism in four dimensions starting from Einstein gravity in five dimensions. He assumed that the five-dimensional space $\mathcal{M}_{5}$ had to be a product of a four-dimensional space-time $M_{4}$ and a circle $S_{1}: \mathcal{M}_{5}=M_{4} \times S_{1}$. It was shown that the zero mode sector of the Kaluza model is equivalent to the four-dimensional theory which describes the Einstein gravity with a four-dimensional general coordinate transformations and the Maxwell theory of electromagnetism with a gauge transformations.

Recently some models with extra dimensions have been proposed to attack the electroweak quantum instability of the Standard Model known as hierarchy problem between the electroweak and gravity scales. It is obviously that the basic idea of the Kaluza-Klein scenario may be applied to any model in Quantum Field Theory. As example, let us consider the simplest case of $(4+\mathrm{d})$-dimensional model of scalar field with the action

$$
S=\int d^{4+d} z \sqrt{-\mathcal{G}}\left[\frac{1}{2}\left(\partial_{M} \Phi\right)^{2}-\frac{m^{2}}{2} \Phi^{2}+\frac{G_{(4+d)}}{4 !} \Phi^{4}\right],
$$

where $\mathcal{G}=\operatorname{det}\left|\mathcal{G}_{M N}\right|, \mathcal{G}_{M N}$ is the metric on $\mathcal{M}_{(4+d)}=M_{4} \times K_{d}, M_{4}$ is pseudo-Euclidean Minkowski space-time, $K_{d}$ is a compact internal $d$-dimensional space with the characteristic size $R$. Let $\Delta_{K_{d}}$ be the Laplace operator on the internal space $K_{d}$, and $Y_{n}(y)$ are ortho-normalized eigenfunctions of the Laplace operator

$$
\Delta_{K_{d}} Y_{n}(y)=-\frac{\lambda_{n}}{R^{2}} Y_{n}(y)
$$

and $n$ is a (multi)index labeling the eigenvalue $\lambda_{n}$ of the eigenfunction $Y_{n}(y)$. A $d$-dimensional torus $T^{d}$ with equal radii $R$ is an especially simple example of the compact internal space of extra dimensions $K_{d}$. The eigenfunctions and eigenvalues in this special case look like

$$
Y_{n}(y)=\frac{1}{\sqrt{V_{d}}} \exp \left(i \sum_{m=1}^{d} n_{m} y^{m} / R\right)
$$




$$
\lambda_{n}=|n|^{2}, \quad|n|^{2}=n_{1}^{2}+n_{2}^{2}+\ldots n_{d}^{2}, \quad n=\left(n_{1}, n_{2}, \ldots, n_{d}\right), \quad-\infty \leq n_{m} \leq \infty,
$$

where $n_{m}$ are integer numbers, $V_{d}=(2 \pi R)^{d}$ is the volume of the torus.

To reduce the multidimensional theory to the effective four-dimensional one we wright a harmonic expansion for the multidimensional field $\Phi(z)$

$$
\Phi(z)=\Phi(x, y)=\sum_{n} \phi^{(n)}(x) Y_{n}(y)
$$

The coefficients $\phi^{(n)}(x)$ of the harmonic expansion (35) are called Kaluza-Klein (KK) excitations or KK modes, and they usually include the zero-mode $\phi^{(0)}(x)$, corresponding to $n=0$ and the eigenvalue $\lambda_{0}=0$. Substitution of the KK mode expansion into action (32) and integration over the internal space $K_{d}$ gives

$$
\begin{gathered}
S=\int d^{4} x \sqrt{-g}\left\{\frac{1}{2}\left(\partial_{\mu} \phi^{(0)}\right)^{2}-\frac{m^{2}}{2}\left(\phi^{(0)}\right)^{2}+\frac{g}{4 !}\left(\phi^{(0)}\right)^{4}+\right. \\
\left.+\sum_{n \neq 0}\left[\frac{1}{2}\left(\partial_{\mu} \phi^{(n)}\right)\left(\partial^{\mu} \phi^{(n)}\right)^{*}-\frac{m_{n}^{2}}{2} \phi^{(n)} \phi^{(n) *}\right]+\frac{g}{4 !}\left(\phi^{(0)}\right)^{2} \sum_{n \neq 0} \phi^{(n)} \phi^{(n) *}\right\}+\ldots
\end{gathered}
$$

For the masses of the KK modes one obtains

$$
m_{n}^{2}=m^{2}+\frac{\lambda_{n}}{R^{2}}
$$

and the coupling constant $g$ of the four-dimensional theory is related to the coupling constant $G_{(4+d)}$ of the initial multidimensional theory by the equation

$$
g=\frac{G_{(4+d)}}{V_{d}}
$$

where $V_{d}$ is the volume of the compact internal space of extra dimensions $K_{d}$. The fundamental coupling constant $G_{(4+d)}$ has dimension [mass] ${ }^{-d}$. So, the four-dimensional coupling constant $g$ is dimensionless one as it should be. Eqs. (37/38) represent the basic relations of Kaluza-Klein scenario. Similar relations take place for other types of multidimensional quantum field theoretical models. From four-dimensional point of view we can interpret each KK mode as a particle with the mass $m_{n}$ given by Eq. (37). We see that in according with Kaluza-Klein scenario any multidimensional field contains an infinite set of KK modes, i.e. an infinite set of four-dimensional particles with increasing masses, which is called the Kaluza-Klein tower. Therefore, an experimental observation of series KK excitations with a characteristic spectrum of the form (37) would be an evidence of the existence of extra dimensions. So far the KK partners of the particles of the Standard Model have not been observed. In the Kaluza-Klein scenario this fact can be explained by a microscopic small size $R$ of extra dimensions $\left(R<10^{-17} \mathrm{~cm}\right)$; in that case the KK excitations may be produced only at super-high energies of the scale $E \sim 1 / R>1 \mathrm{TeV}$. Below this scale only homogeneous zero modes with $n=0$ are accessible ones for an observation in recent high energy experiments. That is why, there is a hope to search the KK excitations at the future LHC and other colliders.

The most recent developments are related with a remarkable idea of "brane world picture" according to which all matter fields (except gravity) are localized on a threedimensional submanifold - brane - embedded in fundamental multidimensional space. In the brane world scenario extra dimensions may have large and even very large size. 
Even though the models with the brane world scenario may rather seem as exotic ones, nevertheless, they provide a base for a nontrivial phenomenological issues related to the fundamental problems in particle physics and cosmology. We refer with a pleasure the interested reader to the excellent review articles [17, 18] and many references therein.

In our recent paper [19] we argued in favour of that the extra dimensions have been observed for a long time in the experiments at very low energies where the nucleonnucleon dynamics has been studied: We have shown that the structure of proton-proton total cross section at very low energies revealed a clear signature of the existence of the extra dimensions. Here we repeat our arguments applying the main issues of Kaluza-Klein approach to our concrete case.

Let us assume that $\mathcal{L}$-particle is related to the first $\mathrm{KK}$ excitation in the diproton system. Using formula (37) for the masses of KK modes, we can calculate the scale (size) $R$ of the compact internal extra space. So, starting from the formula

$$
\sqrt{s_{t h r}}=2 m_{p}+m_{\mathcal{L}}=2 \sqrt{m_{p}^{2}+\frac{1}{R^{2}}}
$$

one obtains

$$
\frac{1}{R}=\sqrt{m_{\mathcal{L}}\left(m_{p}+\frac{1}{4} m_{\mathcal{L}}\right)}=41.481 \mathrm{MeV},
$$

where $m_{p}=938.272 \mathrm{MeV}$ for the proton mass and Eq. (30) for the mass of $\mathcal{L}$-particle have been used. From Eq. (40) it follows

$$
R=24.1 \mathrm{GeV}^{-1}=4.7510^{-13} \mathrm{~cm} .
$$

It should be emphasized a remarkable fact: the size (41) just corresponds to the scale of distances where the strong Yukawa forces in strength come down to the electromagnetic forces

$$
g_{\text {eff }}=g_{\pi N N} \exp \left(-m_{\pi} R\right) \sim 0.5, \quad\left(g_{\pi N N}^{2} / 4 \pi=14.6\right) .
$$

On the other hand, for the fundamental mass scale with account of size (41) in the case $d=6$ we find $[19$

$$
\left.M \sim R^{-1}\left(\frac{M_{P l}}{R^{-1}}\right)^{2 /(d+2)}\right|_{d=6} \sim 5 \mathrm{TeV} .
$$

Mass scale (42) is just the scale accepted in the Standard Model, and this is an interesting observation as well.

Going further on, let us build the Kaluza-Klein tower of KK excitations by the formula ${ }^{2}$

$$
M_{n}=2 \sqrt{m_{p}^{2}+\frac{n^{2}}{R^{2}}}, \quad(n=1,2,3, \ldots)
$$

and compare it with the observed irregularities in the spectrum of mass of the diproton system. The result of the comparison is shown in Table 2. As it is seen from the Table 2, there is a quite remarkable correspondence of the Kaluza-Klein picture with the experiment. It is pleased for me to tell about it here at this Conference because the main part from the list of diproton resonances has been discovered and observed in Dubna.

\footnotetext{
${ }^{2}$ Similar formula has been discussed in the literature [28] but with a different physical interpretation.
} 
Now, let us suppose that effective bosons $B_{n}$ with the masses $m_{n}=n / R$ related to KK-excitations of a proton may have an effective Yukawa-type interaction with the fermions

$$
L_{e f f}=g_{e f f} \bar{\psi}_{f} O \psi_{f} B_{n}
$$

where $f$ denotes some fermion, for example lepton or quark.

Table 2. Kaluza-Klein tower of KK excitations of diproton system.

\begin{tabular}{|c|c|c|c|}
\hline $\mathrm{n}$ & $M_{n}(\mathrm{MeV})$ & $M_{e x p}^{p p}(M e V)$ & Refs. \\
\hline 1 & 1878.38 & $1877.5 \pm 0.5$ & [20] \\
\hline 2 & 1883.87 & $1886 \pm 1$ & [14] \\
\hline 3 & 1892.98 & $1898 \pm 1$ & {$[14]$} \\
\hline 4 & 1905.66 & $1904 \pm 2$ & {$[21]$} \\
\hline \multirow[t]{2}{*}{5} & 1921.84 & $1916 \pm 2$ & {$[14]$} \\
\hline & & $1926 \pm 2$ & [21] \\
\hline \multirow{5}{*}{6} & & $1937 \pm 2$ & [14] \\
\hline & & $1942 \pm 2$ & [21] \\
\hline & 1941.44 & $1945 \pm 2.5$ & [15] \\
\hline & & $1955 \pm 2$ & [15] \\
\hline & & $1956 \pm 3$ & {$[22]$} \\
\hline \multirow[t]{2}{*}{7} & 1964.35 & $1965 \pm 2$ & {$[14]$} \\
\hline & & $1969 \pm 2$ & [24] \\
\hline \multirow[t]{2}{*}{8} & 1990.46 & $1980 \pm 2$ & [14] \\
\hline & & $1999 \pm 2$ & [14] \\
\hline 9 & 2019.63 & $2017 \pm 3$ & {$[14]$} \\
\hline \multirow{3}{*}{10} & & $2035 \pm 8$ & {$[22]$} \\
\hline & 2051.75 & $2046 \pm 3$ & [14] \\
\hline & & $2050 \pm 3.2$ & [25] \\
\hline 11 & 2086.68 & $2087 \pm 3$ & [14] \\
\hline \multirow{3}{*}{12} & & $2120 \pm 3.2$ & [25] \\
\hline & 2124.27 & $2121 \pm 3$ & [26] \\
\hline & & $2129 \pm 5$ & [14] \\
\hline \multirow{3}{*}{13} & & $2140 \pm 9$ & [22] \\
\hline & 2164.39 & $2150 \pm 12.6$ & [25] \\
\hline & & $2172 \pm 5$ & [14] \\
\hline \multirow{3}{*}{14} & & $2192 \pm 3$ & [26] \\
\hline & 2206.91 & 2217 & [16] \\
\hline & & 2220 & [23] \\
\hline \multirow[t]{2}{*}{15} & 2251.67 & $2238 \pm 3$ & {$[14]$} \\
\hline & & $2240 \pm 5$ & [26] \\
\hline 16 & 2298.57 & $2282 \pm 4$ & {$[14,27]$} \\
\hline 17 & 2347.45 & 2350 & [23] \\
\hline
\end{tabular}

If $m_{n}>2 m_{f}$ then effective bosons may decay into fermion-antifrermion pair. For the partial width of such decay in the lowest order over coupling constant we have

$$
\Gamma_{n}=\frac{\alpha_{e f f} m_{n}}{2} F_{O}\left(x_{n}^{2}\right),
$$


where $\alpha_{\text {eff }}=g_{\text {eff }}^{2} / 4 \pi, x_{n}^{2}=m_{f}^{2} / m_{n}^{2}, F_{O}\left(x^{2}\right)=\left(1-4 x^{2}\right)^{3 / 2}$ for $O=1$ and $F_{O}\left(x^{2}\right)=$ $\left(1-4 x^{2}\right)^{1 / 2}$ for $O=\gamma_{5}$. In that case one obtains an estimation

$$
\Gamma_{n} \sim n \cdot 0.4 \mathrm{MeV}
$$

It's clear from the physics under consideration that a life time of the diproton resonances will be defined by the decays of effective bosons $B_{n}$. This is a remarkable fact that crude estimation (46) is in a good agreement with an experiment and gives an explanation of (super)narrowness of dibaryons peaks. Moreover, estimation (46) shows that the larger the dibaryon mass is, the larger is the width of the dibaryon.

Here we have concerned the simplest model where the protons were considered as a scalar particles. It is well known that account of fermionic degrees of freedom may result the nontrivial problems related to both the index and the kernel of Dirac operator on a generic compact manifold. However, since the kernel of Dirac operator is equal to the kernel of its square, we can say with confidence that account of fermionic degrees of freedom for a proton will not change our main conclusion.

Certainly, we have also considered here the simplest case of Kaluza-Klein picture: The built KK-tower corresponds to either one-dimensional compact extra space or ddimensional equal radii torus with the constraint

$$
n=\sqrt{n_{1}^{2}+n_{2}^{2}+\ldots n_{d}^{2}}=1,2,3, \ldots,
$$

where $n_{i}(i=1, \ldots, d)$ are integer numbers. The constraint (47) corresponds to the special (Diophantus!) selection of the states. It's clear that in general case of generic extra compact manifold we would have a significantly more wealthy spectrum of KK-excitations. One could imagine that there exist such extra compact manifold with a suitable geometry where KK-excitations of a few input fundamental entities (proton, electron, photon, etc.) would provide the experimentally observed spectrum of all particles, their resonances and nuclei states. As we hope, it would be possible to find in this way the global solution of the Spectral Problem. Anyhow, we believe that such perfect extra compact manifold with a beautiful geometry and its good-looking shapes exist.

\section{Conclusion}

Investigating the three-body forces open many new pages in the study of fundamental dynamics of particles and nuclei:

- Three-body forces define the dynamics of one-particle inclusive reactions.

- We have to take into account a contribution of three-body forces in scattering from deuteron and, in general, from nuclei.

- New scaling characteristics in shadow dynamics in scattering from deuteron have been established by account of three-body forces.

- Introduction of three-body forces resulted the discovery of global structure of (anti)proton-proton total cross sections.

- Investigating the three-body forces allowed us to predict a new particle $(\mathcal{L}$-particle), describing a new scale of internucleon distances, where strong Yukawa forces compared with electromagnetic ones. 
We would like to emphasize that multidimensional (six-dimensional) space is a natural space to describe the properties of three-body forces. Geniusly simple formula provided by Kaluza-Klein approach so accurately described the mass spectrum of diproton system, and certainly it was not an accidental coincidence. This means that the existence of the extra dimensions was experimentally proved in the experiments at very low energies where the nucleon-nucleon dynamics had been studied, but we did not understand it. However, now it seems we understand it.

\section{Acknowledgements}

It is my great pleasure to express thanks to the Organizing Committee for the kind invitation to attend the XXXII International Symposium on Multiparticle Dynamics. I would like to especially thank A.N. Sissakian, G. Kozlov and all local organizers from Dubna for excellently organized Conference.

\section{References}

[1] A.A. Arkhipov, Sov. J. Theor. Math. Phys. 74, 69 (1988); ibid 83, 247 (1990).

[2] A.A. Arkhipov, V.I. Savrin, Sov. J. Theor. Math. Phys. 16, 328 (1973).

[3] A.A. Arkhipov, V.I. Savrin, Sov. J. Theor. Math. Phys. 49, 3 (1981).

[4] A.A. Arkhipov, Rep. on Math. Phys. 20, 303 (1984).

[5] A.A. Arkhipov, V.I. Savrin, Sov. J. Theor. Math. Phys. 24, 78 (1975).

[6] A.A. Arkhipov, Sov. J. Theor. Math. Phys. 49, 320 (1981).

[7] A.A. Arkhipov, What Can we Learn from the Study of Single Diffractive Dissociation at High Energies? - in Proceedings of VIIIth Blois Workshop on Elastic and Diffractive Scattering, Protvino, Russia, June 28-July 2, 1999, World Scientific, Singapore, 2000, pp. 109-118; REPORT IHEP 99-43, Protvino, 1999; e-print hep-ph/9909531.

[8] A.A. Arkhipov, Three-Body Forces, Single Diffraction Dissociation and Shadow Corrections in Hadron-Deuteron Total Cross Sections, preprint IHEP 2000-59, Protvino, 2000; e-print hep-ph/0012349; in Proceedings of XVth Workshop on High Energy Physics and Quantum Field Theory, Tver, Russia, September 7-13, 2000, eds. M. Dubinin, V. Savrin, Institute of Nuclear Physics, Moscow State University, Russia, 2001, pp. 241-257.

[9] A.A. Arkhipov,DIFFRACTION 2000: New Scaling Laws in Shadow Dynamics, Nucl. Phys. B (Proc. Suppl.) 99A, 72 (2001).

[10] A.A. Arkhipov, On Global Structure of Hadronic Total Cross Sections, preprint IHEP 99-45, Protvino, 1999; e-print hep-ph/9911533.

[11] F. Schmidt-Kaler et al., Phys. Rev. Lett. 70, 2261 (1993). 
[12] A.A. Arkhipov, Proton-Proton Total Cross Sections from the Window of Cosmic Ray Experiments, preprint IHEP 2001-23, Protvino, 2001; e-print hep-ph/0108118; in Proceedings of IXth Blois Workshop on Elastic and Diffractive Scattering, Pruhonice near Prague, Czech Republic, June 9-15, 2001, eds. V. Kundrat, P. Zavada, Institute of Physics, Prague, Czech Republic, 2002, pp. 293-304.

[13] The computer readable files of data available at http://pdg.lbl.gov and also accessible in IHEP COMPAS database at http://www.ihep.su:8001/ppds.html.

[14] Yu.A. Troyan, V.N. Pechenov, Sov. J. Yad. Phys. 56, 191 (1993); Yu.A. Troyan, Sov. J. Physics of Element. Part. and Atomic Nuclei 24, 683 (1993).

[15] B. Tatischeff et al., Phys. Rev. C45 2005 (1992).

[16] A.A. Arkhipov, On a Manifestation of Dibaryon Resonances in the Structure of Proton-Proton Total Cross Section at Low Energies, preprint IHEP 2001-44, Protvino, 2001; e-print hep-ph/0110399; in Proceedings of the Ninth International Conference on Hadron Spectroscopy, Protvino, Russia, 25 August-1 September, 2001, eds. D. Amelin, A.M. Zaitsev, Melville, New York, 2002, AIP Conference Proceedings, vol. 619, pp.771-776.

[17] V.A. Rubakov, Large and infinite extra dimensions, Sov. J. Uspekhi 171, 913 (2001); e-print hep-ph/0104152.

[18] Yu.A. Kubyshin, Models with Extra Dimensions and Their Phenomenology, e-print hep-ph/0111027.

[19] A.A. Arkhipov, $\mathcal{L}$-Particle and Kaluza-KLein World, e-print hep-ph/0208263.

[20] B.M. Abramov et al., Sov. J. Yad. Phys. 57, 850 (1994).

[21] L.V. Filkov et al., e-prints nucl-th/0009044 hep-ex/0006029.

[22] V.P. Andreev et al., Z. Phys. A327 363 (1987).

[23] Y. Ohashi et al., Phys. Rev. C36 2432 (1987).

[24] B. Tatischeff et al., Z. Phys. A328 147 (1987).

[25] B. Tatischeff et al., Phys. Rev. C59 1878 (1999).

[26] B. Tatischeff et al., Phys. Rev. C36 1995 (1987).

[27] B. Tatischeff et al., in Proceedings of the Xth International Seminar on High Energy Physics Problems "Relativistic Nuclear Physics\&Quantum Chromodynamics", Dubna, 24-29 September, 1990, USSR, eds. A.M. Baldin, V.V. Burov, L.P. Kaptari, World Scientific, 1990, p. 177; in Proceedings of the XIIth International Seminar on High Energy Physics Problems "Relativistic Nuclear Physics\&Quantum Chromodynamics", Vol. II, Dubna, 1997, p. 62.

[28] F.A. Gareev, G.S. Kazacha, Yu.L. Ratis, Sov. J. Physics of Element. Part. and Atomic Nuclei 27, 97 (1996). 


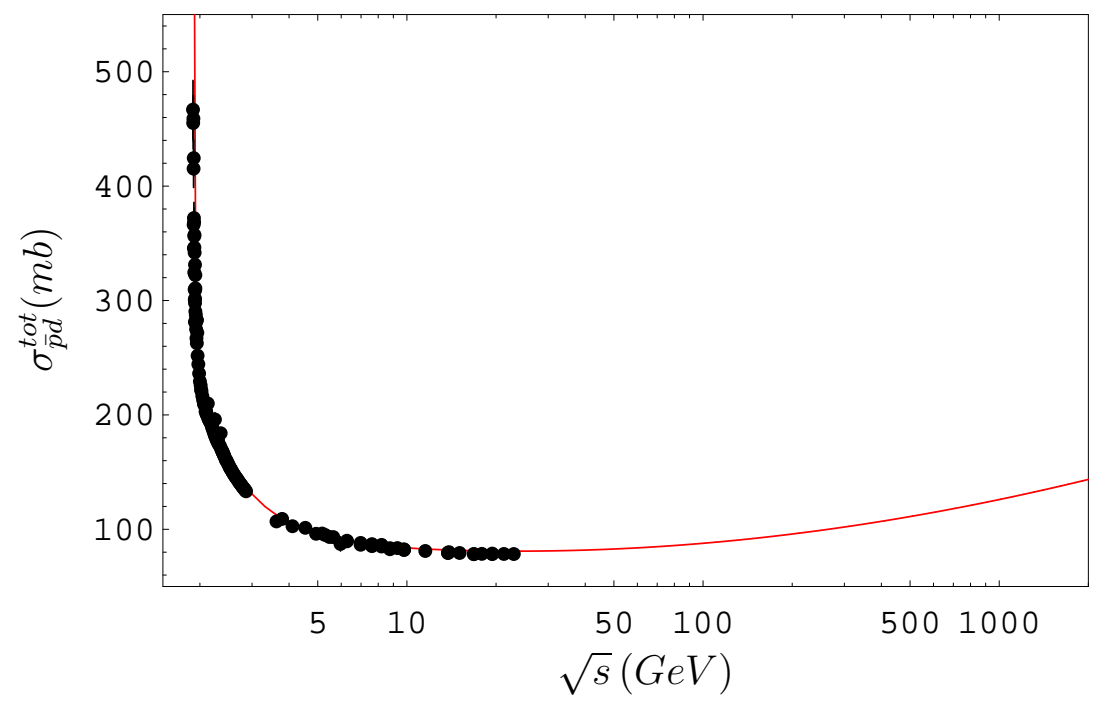

Figure 1: The total antiproton-deuteron cross-section compared with the theory. Statistical and systematic errors added in quadrature.

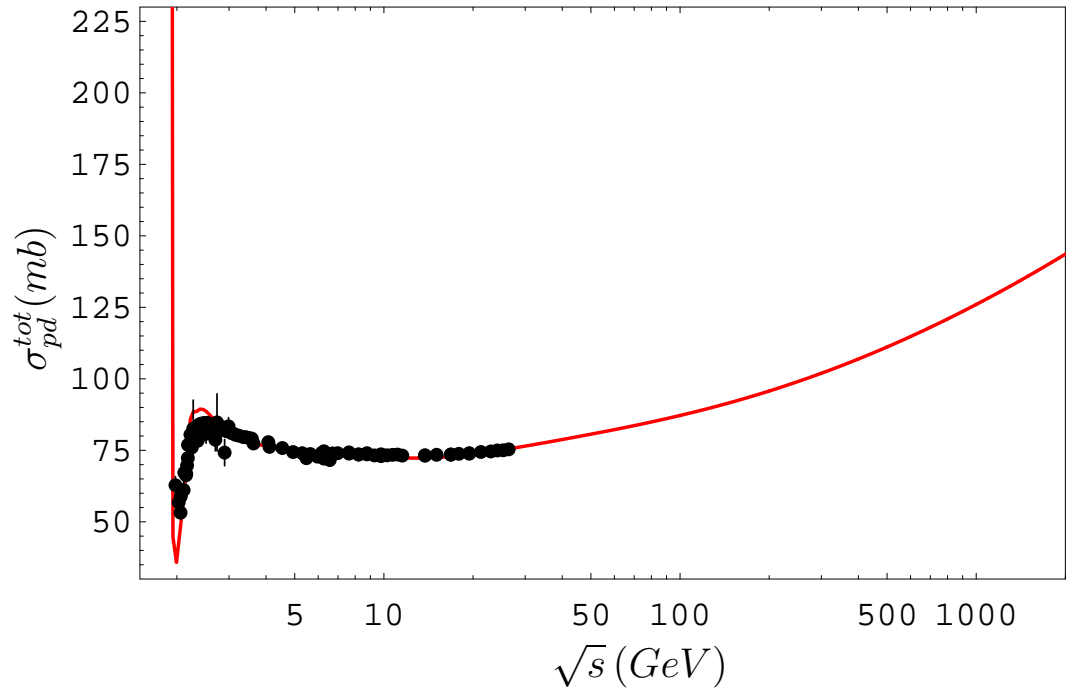

Figure 2: The total proton-deuteron cross-section compared with the theory without any free parameters. Statistical and systematic errors added in quadrature. 


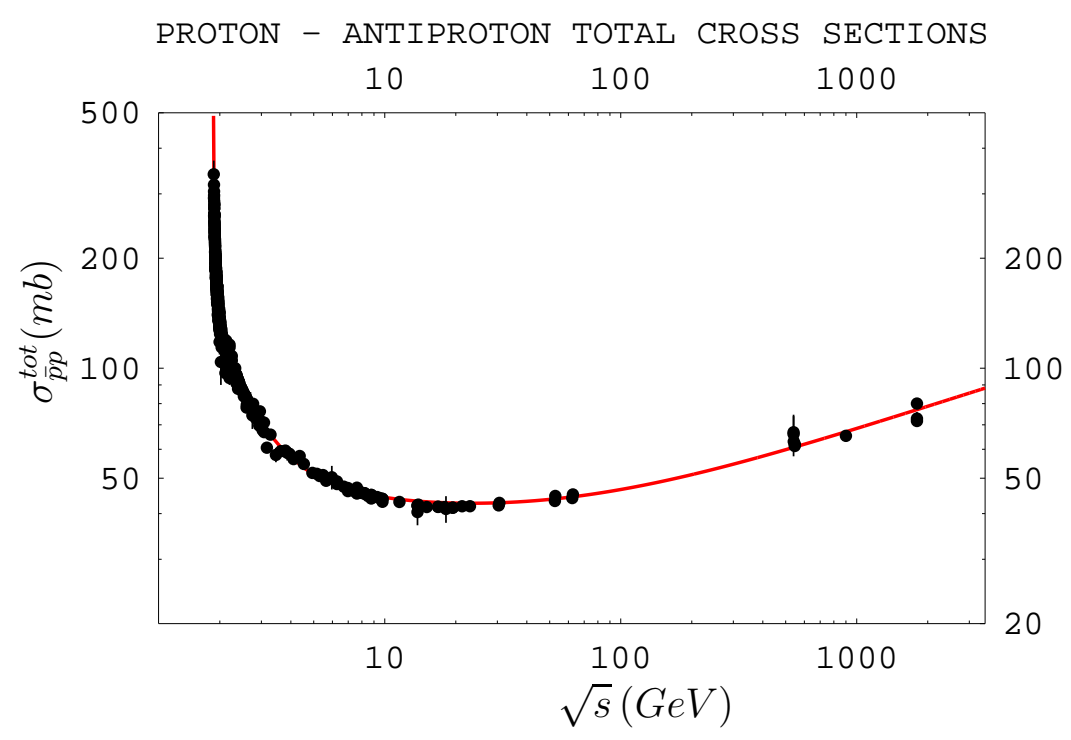

Figure 3: The proton-antiproton total cross sections versus $\sqrt{s}$ compared with the theory. Solid line represents our fit to the data. Statistical and systematic errors added in quadrature.

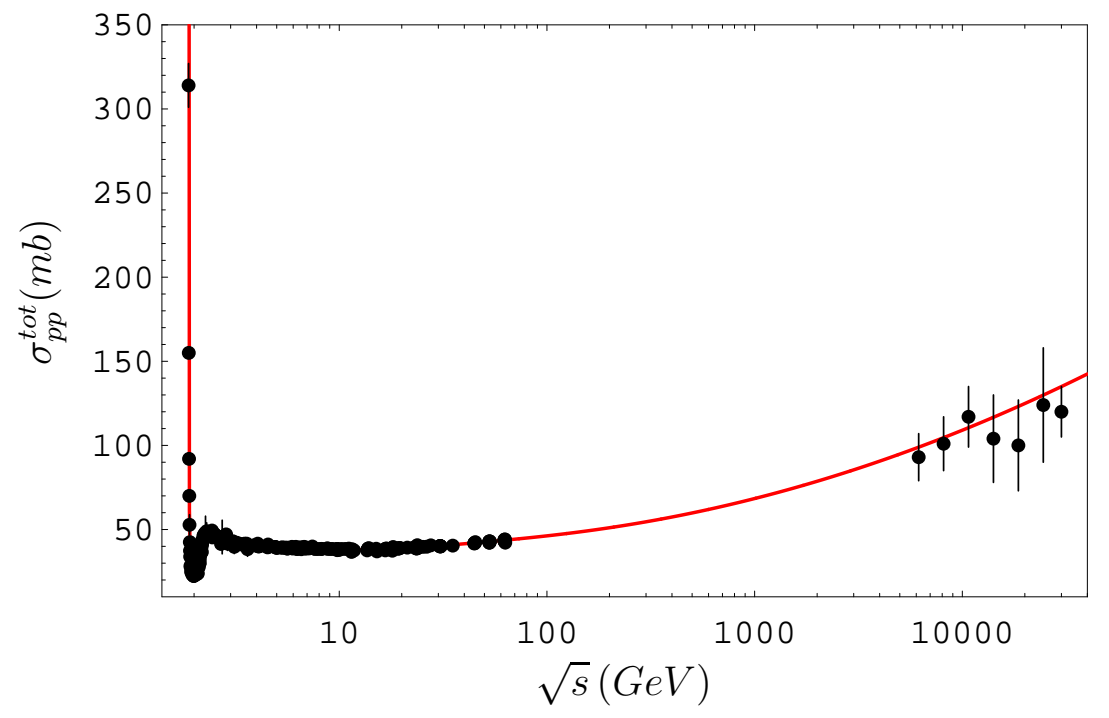

Figure 4: The proton-proton total cross-section versus $\sqrt{s}$ with the cosmic rays data points from Akeno Observatory and Fly's Eye Collaboration. Solid line corresponds to our theory predictions. 


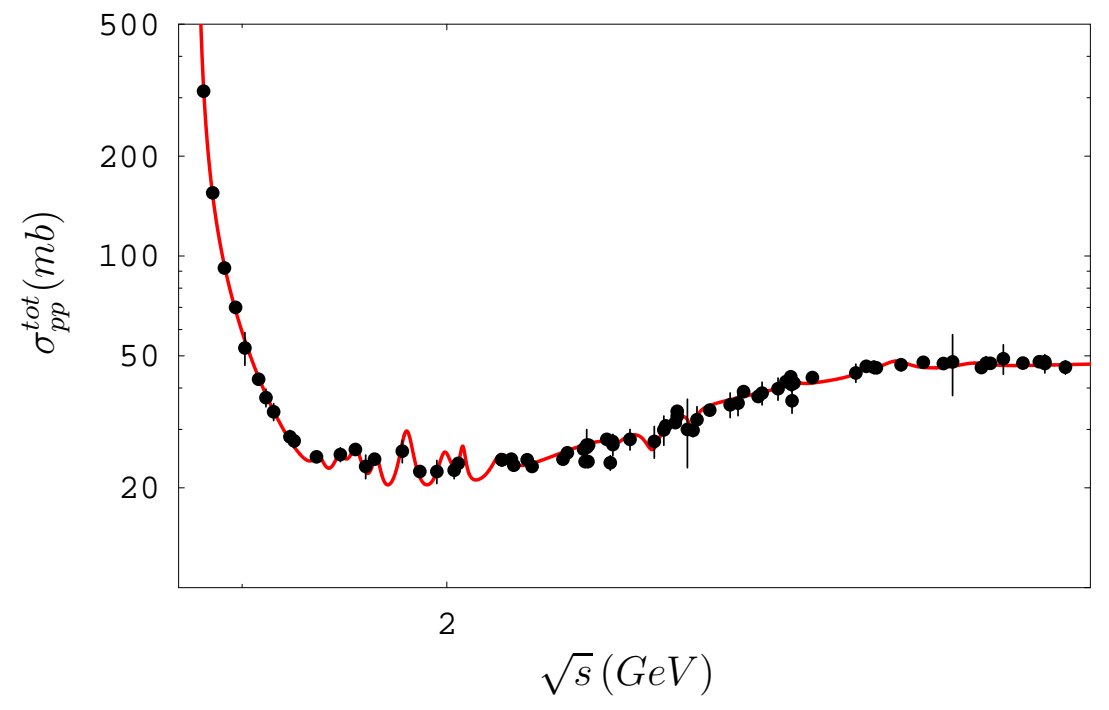

Figure 5: The proton-proton total cross-section versus $\sqrt{s}$ at low energies. Solid line corresponds to our theory predictions.

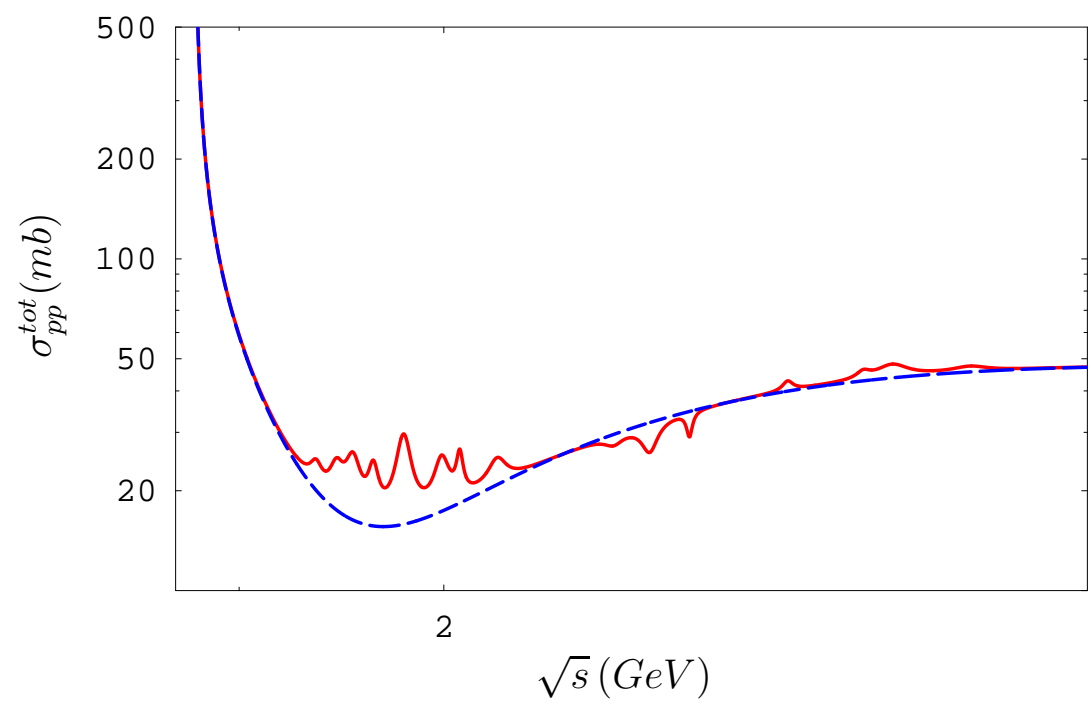

Figure 6: The resonance structure for the proton-proton total cross-section versus $\sqrt{s}$ at low energies. Solid line is our theory predictions. Dashed line corresponds to the "background" where all resonances are switched off. 